Subscriber access provided by Caltech Library

\title{
Article
}

\section{Mechanistic Studies of the Photoinduced Quinone Trimethyl Lock Decaging Process}

Clinton J Regan, David P. Walton, Oliver S Shafaat, and Dennis A. Dougherty

J. Am. Chem. Soc., Just Accepted Manuscript • DOI: 10.1021/jacs.6b12007 • Publication Date (Web): 15 Feb 2017

Downloaded from http://pubs.acs.org on February 21, 2017

\section{Just Accepted}

"Just Accepted" manuscripts have been peer-reviewed and accepted for publication. They are posted online prior to technical editing, formatting for publication and author proofing. The American Chemical Society provides "Just Accepted" as a free service to the research community to expedite the dissemination of scientific material as soon as possible after acceptance. "Just Accepted" manuscripts appear in full in PDF format accompanied by an HTML abstract. "Just Accepted" manuscripts have been fully peer reviewed, but should not be considered the official version of record. They are accessible to all readers and citable by the Digital Object Identifier (DOI®). "Just Accepted" is an optional service offered to authors. Therefore, the "Just Accepted" Web site may not include all articles that will be published in the journal. After a manuscript is technically edited and formatted, it will be removed from the "Just Accepted" Web site and published as an ASAP article. Note that technical editing may introduce minor changes to the manuscript text and/or graphics which could affect content, and all legal disclaimers and ethical guidelines that apply to the journal pertain. ACS cannot be held responsible for errors or consequences arising from the use of information contained in these "Just Accepted" manuscripts. 


\title{
Mechanistic Studies of the Photoinduced Quinone Trimethyl Lock Decaging Process
}

\author{
Clinton J. Regan, David P. Walton, Oliver S. Shafaat, and Dennis A. Dougherty* \\ Division of Chemistry and Chemical Engineering, California Institute of Technology, Pasadena, CA 91125 USA
}

\begin{abstract}
Mechanistic studies of a general reaction that decages a wide range of substrates on exposure to visible light are described. The reaction involves a photochemically initiated reduction of a quinone mediated by an appended thioether. After reduction, a trimethyl lock system incorporated into the quinone leads to thermal decaging. The reaction could be viewed as an electron-transfer initiated reduction of the quinone or as a hydrogen abstraction - Norrish Type II - reaction. Product analysis, kinetic isotope effects, stereochemical labeling, radical clock, and transient absorption studies support the electron transfer mechanism. The differing reactivities of the singlet and triplet states are determined, and the ways in which this process deviates from typical quinone photochemistry are discussed. The mechanism suggests strategies for extending the reaction to longer wavelengths that would be of interest for applications in chemical biology and in a therapeutic setting.
\end{abstract}

\section{Introduction and Synthesis}

We recently described a new class of compounds that undergo photochemical decaging of a wide range of substrates at wavelengths as long as $600 \mathrm{~nm}$. ${ }^{1}$ Such compounds could find use as chemical biology tools, and in a therapeutic setting, where longer wavelengths lead to deeper tissue penetration. In an effort to maximize decaging efficiency and to provide insights into possible strategies for extending the photoreactivity to longer wavelengths, we have conducted extensive mechanistic studies of the photoreaction. Herein we describe those mechanistic studies and the design strategies they suggest.

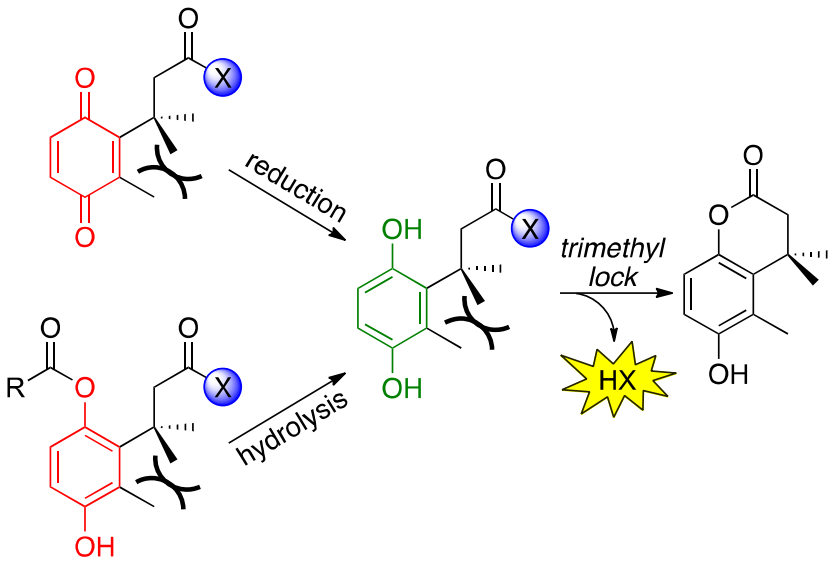

Figure 1. Variants of the trimethyl lock decaging process.

The initial approach sought to take a known chemical decaging process and design systems that could be phototriggered. Figure 1 shows two variants of the well-established trimethyl lock system. ${ }^{2-4}$ Either reducing a quinone or revealing a phenol produces a nucleophile that can exploit the remarkable rate enhancements associated with the trimethyl lock system, releasing $\mathbf{H X}$ as a generic alcohol, amine, thiol, or phosphate. Of course, deprotection of the phenol can be accomplished photochemically using established caging groups, ${ }^{5,6}$ but this approach does not lead naturally to longer wavelength systems.

Scheme 1. Products of photolysis of 1 at $420 \mathrm{~nm}$.

1

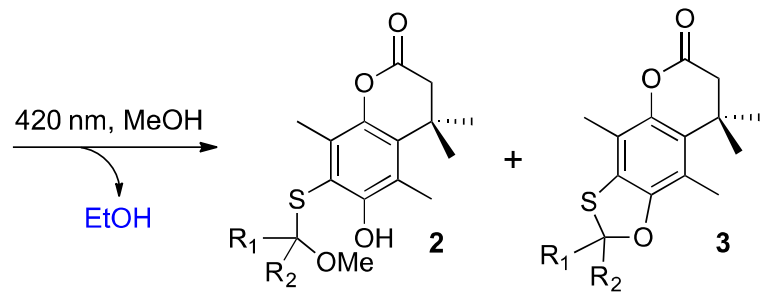

1
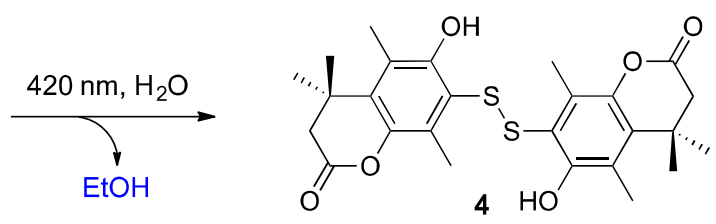

$1 \mathrm{j}$

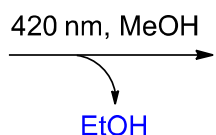



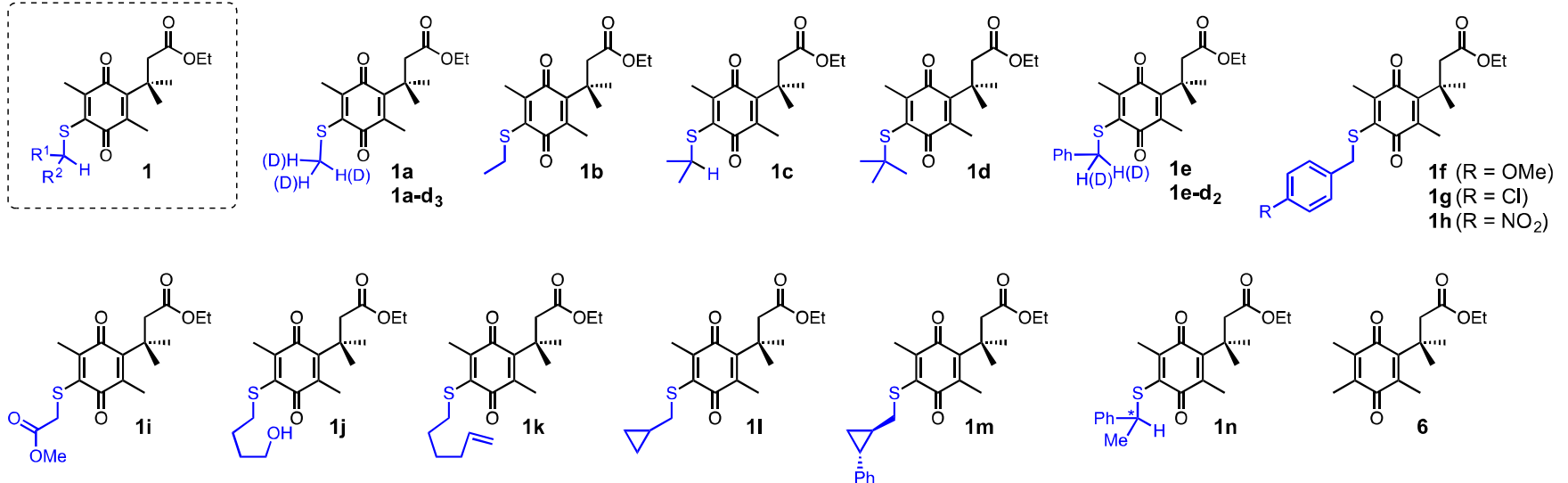

Figure 2. Photoinduced quinone trimethyl lock derivatives discussed in this work.

With a goal of creating a photochemically triggered trimethyl lock system, we considered compound 1 (Figure 2). The bimolecular photoreduction of quinones by sulfides has been reported, but, in general, the reaction has not been extensively studied. $^{7,8}$ The process is believed to be initiated by an electron transfer (ET) followed by a crucial C-H oxidation, similar to photoreduction by amines. ${ }^{9-12}$ Intramolecular variants are known, ${ }^{13,14}$ but for these reactions a direct hydrogen abstraction (Norrish Type II-like) process cannot be ruled out. For the present purposes, we sought to employ an ET mechanism, as this seemed better suited for longer wavelengths. Although most quinone photoreductions involve amines, ${ }^{15-22}$ we chose a sulfide as the potential electron donor in our initial design. We anticipated a more facile synthesis of the desired systems, more favorable redox properties, and perhaps greater stability in air and in a biological system. The synthesis of $\mathbf{1}$ is efficient and permits a wide variety of sulfide substituents to be introduced in the last step (Figure 2). A representative UV/Vis spectrum is shown in Figure 3 for the methyl derivative (1a). Notably, a broad absorption band is observed at approximately $413 \mathrm{~nm}$; the relevant data for this band $\left(\lambda_{\max }\right.$ and $\left.\varepsilon\right)$ for key substrates are reported in Table 1. We have been unable to observe luminescence from 1a, either in fluid media at room temperature or at $77 \mathrm{~K}$ in a frozen matrix, as is typical of quinones. ${ }^{23-30}$

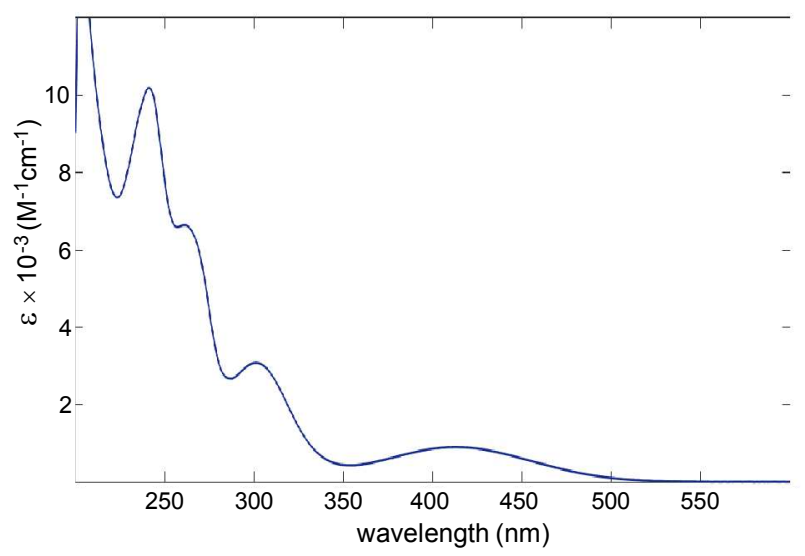

Figure 3. Absorbance spectrum of 1a in methanol.

\section{Steady-State Photolysis}

Photolysis of 1a with, for example, a 420 or $455 \mathrm{~nm}$ LED, in air-equilibrated methanol leads to the clean formation of thioacetal 2a (Scheme 1), as observed by ${ }^{1} \mathrm{H}$ NMR of the crude product. When the reaction is carried out in deuterated solvent, ethanol can also be detected in the reaction mixture as the exclusive byproduct. For some variants of $\mathbf{1}$, other reaction products are observed in the crude and can be isolated by silica gel chromatography. Compound $\mathbf{3}$ is presumably produced by intramolecular capture of the species that is trapped by methanol. Photolysis in water (pure or buffered to $\mathrm{pH} 7.5$ ) also releases the caged alcohol and produces the disulfide 4, presumably via a thiohemiacetal intermediate. Both reactions are very clean; quantum yields will be discussed below. In other solvents such as acetonitrile, benzene, or hexane, the reaction is slower and produces a complex mixture of products.

Compound $\mathbf{1} \mathbf{j}$ contains a tethered alcohol, and upon photolysis in methanol it cleanly produces both $\mathbf{2} \mathbf{j}$ and the expected cyclic product 5 in a 1:4 ratio (Scheme 1). Photolysis of $\mathbf{1 j}$ in acetonitrile or benzene does produce the cyclic product, but there are also other intractable products. Compounds $\mathbf{1 h}$ and 1i, both possessing electron-withdrawing groups, are peculiar in that they produce decomposition products upon photolysis in methanol, and display visible absorption bands that are weak and blue-shifted (Table 1). It is clear that some of the decomposition products have not undergone trimethyl lock lactonization, suggesting that unmasking of the phenol has not occurred. Compound 1d lacks the necessary $\gamma$-hydrogen on sulfur and is found to be nonreactive to photolysis at $420 \mathrm{~nm}$. By comparison, compound 6 (Figure 2) lacks a sulfur substituent altogether, and it is found to undergo $\gamma$-hydrogen abstraction from the trimethyl lock side chain. Similar processes have been reported previously. ${ }^{7,31-37}$

\section{Mechanistic Studies}

Most of our mechanistic studies have been conducted in methanol, where the reaction is clean and solubility is not an issue. We will examine the mechanism by working backwards from the final product. It is clear that the actual ring closure of the trimethyl lock and the release of the caged compound is the final and slowest step of the process. One could have imagined that an initial photochemical ET from the sulfide to the quinone would build enough negative charge on the quinone oxygens such that a trimethyl lock closure could occur before 
further reduction of the quinone, ${ }^{18}$ but that is not the case. In deuterated methanol, the hydroquinone ( 7 , Scheme 2$)$ can be directly observed by ${ }^{1} \mathrm{H}$ NMR prior to trimethyl lock ring closure. In aqueous systems the ring closure is rapid for an alcohol leaving group, but not for an amine leaving group, again allowing the hydroquinone to be observed prior to trimethyl lock closure. ${ }^{1}$ These results could be anticipated based on known trimethyl lock rates. ${ }^{2}$

The mechanistic issue then becomes the conversion of quinone 1 to the methanol adduct hydroquinone, 7. The requirement for a solvent capture step implicates zwitterion $\mathbf{8}$ as the likely precursor to 7 . Conceptually, the conversion of $\mathbf{1}$ to $\mathbf{8}$ then requires reduction of the quinone by two electrons and the shift of a proton from the carbon attached to the sulfur to the quinone oxygen. The matter in question is whether the reaction proceeds through ionic intermediates, similar to bimolecular photoreductions by sulfides and amines, or through a traditional radical mechanism similar to the Norrish II (Scheme 2). To keep the semantics straight, we will use the term hydrogenshift as noncommittal regarding all steps in the process $\mathbf{1} \rightarrow \mathbf{8}$.

Scheme 2. Potential intermediates and pathways in the photolysis of 1 .
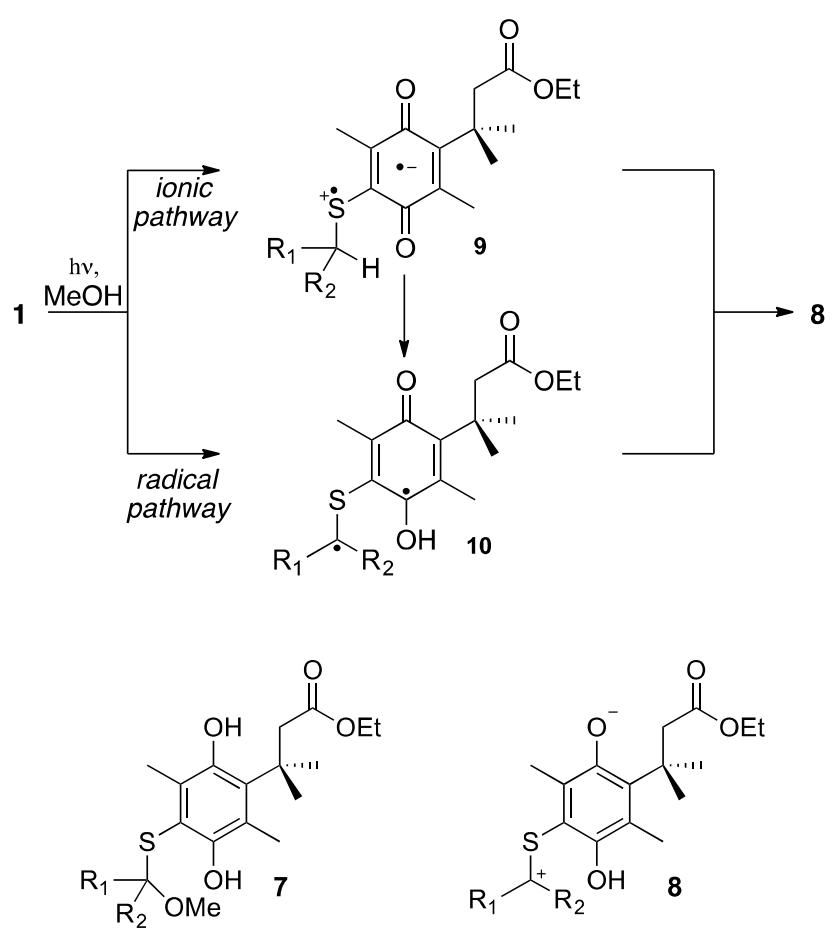

We have applied a number of mechanistic tools to this reaction. First, the influence of sulfide substituents on the quantum yield for product formation was probed. Using a ferrioxalate actinometer $^{38}$ we have determined the quantum yield $(\Phi)$ for the conversion of $\mathbf{1}$ in degassed methanol solutions, and the results are summarized in Table 1 . The effect of added oxygen on the quantum yield is generally small and will be discussed further below. There is a trend of $i$-propyl (1c) $>$ ethyl (1b) $>$ methyl (1a) in relative quantum yield, although the effect is not large. A benzyl substituent (1e) shows the largest effect, with a $>5$-fold increase in quantum yield. These trends would be consistent with either radical or cationic character building up on the substituted carbon. However, substituted benzyl compounds (1f,1g) do not follow a simple trend, and we note again that the $p$-nitrobenzyl substrate $\mathbf{1 h}$ produces a complex mixture of products.

We next considered the role of the hydrogen shift on the overall process. There is an isotope effect $\left(\Phi_{H} / \Phi_{D}\right)$ on the quantum yield for quinone disappearance. A value of 4.0 is obtained for the methyl compound (1a vs. 1a-d $\mathbf{d}_{3}$ ), and 2.5 for the benzyl compound (1e vs. 1e-d $\mathbf{d}_{2}$ ) (Table 1). These observations highlight the critical role of the hydrogen-shift in the overall process.

Table 1. Spectroscopic and photolysis data for 1 in $\mathrm{MeOH}^{a}$

$\begin{array}{lccccccccc} & \lambda_{\max }^{b} & \varepsilon^{b} & \boldsymbol{\Phi}^{c} & \tau^{d} & \Phi^{S^{e}} & \boldsymbol{\Phi}^{T^{e}} & \phi_{i s c}^{\mathbf{f}} & \phi_{r x n}^{T^{g}} & \mathbf{k}_{r x n}^{T^{h}} \\ \mathbf{1} & (\mathrm{nm}) & \left(\mathrm{M}^{-1} \mathrm{~cm}^{-1}\right) & (\%) & (\mathrm{ns}) & (\%) & (\%) & (\%) & (\%) & \left(10^{5} \mathrm{~s}^{-1}\right) \\ \mathbf{1 a} & 413 & 903 & 1.2 & 930 & 1.0 & 0.2 & 0.9 & 21 & 2.3 \\ \mathbf{1 a}_{\mathbf{1}} \mathbf{d}_{\mathbf{3}} & 413 & 923 & 0.3 & 1070 & 0.27 & 0.03 & 10 & 0.3 & 0.03 \\ \mathbf{1 b} & 414 & 1005 & 1.7 & 750 & 1.5 & 0.2 & 1.3 & 15 & 2.0 \\ \mathbf{1 c} & 411 & 927 & 2.2 & 543 & 1.6 & 0.6 & 2.8 & 21 & 3.9 \\ \mathbf{1 e} & 413 & 951 & 6.3 & 143 & 3.0 & 3.3 & 4.5 & 73 & 51 \\ \mathbf{1 e - d _ { 2 }} & 412 & 944 & 2.5 & 577 & 1.0 & 1.5 & 3.5 & 42 & 7.3 \\ \mathbf{1 f} & 410 & 953 & 3.1 & 600 & - & - & - & 44 & 7.3 \\ \mathbf{1 g} & 409 & 832 & 5.2 & 180 & - & - & - & 89 & 49 \\ \mathbf{1 h} & 396 & 709 & 0.6 & - & - & - & - & - & - \\ \mathbf{1 i} & 394 & 604 & 0.6 & - & - & - & - & - & -\end{array}$

${ }^{a}$ All quantum yields are reported at $420 \mathrm{~nm}$ relative to ferrioxalate actinometer with a standard deviation of $<10 \%$. ${ }^{b}$ Longest wavelength absorption band in air-equilibrated methanol. ${ }^{c}$ Quantum yield for disappearance of $1 .{ }^{d}$ Lifetime of the transient observed at $480 \mathrm{~nm}$ upon pulsed laser irradiation at $355 \mathrm{~nm}$ in degassed methanol. ${ }^{e}$ Quantum yield for disappearance of $\mathbf{1}$ through the singlet (S) and triplet (T) pathways; ref to Eq. 1. ${ }^{f}$ Quantum efficiency of intersystem crossing for $1 .{ }^{g}$ Minimum value of the quantum efficiency for disappearance of $\mathbf{1}$ from the sensitized state. ${ }^{h}$ Rate constant for reaction from the sensitized state.

Either a radical or ionic mechanism could potentially generate biradical 10 (Scheme 2), with the former being a conventional Norrish II reaction. To probe for the intermediacy of $\mathbf{1 0}$, we incorporated radical clocks into the system, preparing the 5hexenyl (1k), cyclopropylmethyl (11), and 2phenylcyclopropylmethyl (1m) derivatives. These are standard probes that have been used successfully in conventional Norrish II reactions. ${ }^{39-43}$ For both $\mathbf{1 k}$ and $\mathbf{1 1}$, no radical rearrangement is seen; the products $\mathbf{2}$ and $\mathbf{3}$ are cleanly produced. The phenylcyclopropyl clock shows a very fast intrinsic ring opening rate of $10^{11} \mathrm{~s}^{-1} \cdot{ }^{39,44}$ Photolysis of $1 \mathrm{~m}$ produces a $20 \%$ yield of the expected methanol-trapping product with the phenylcyclopropyl ring still intact. The remaining material is a mixture of products that has not been fully characterized. While it is likely true that the sulfur in our system perturbs the radical rearrangements studied here, it seems safe to conclude that if a biradical such as $\mathbf{1 0}$ is directly formed in this system, it has a very short lifetime - much shorter than a typical Norrish II biradical. ${ }^{42}$ An especially telling probe of the role of the hydrogen shift was provided by a stereochemical test. Enantiomerically pure phenethyl derivative 1n was prepared with $>95 \%$ ee as determined by chiral HPLC (Supporting Information). Upon photolysis to $75 \%$ conversion, recovered starting material showed no racemization within the detection limits of the method. As such, we conclude that the hydrogen shift is effectively irreversible, and that nonproductive decay 
of the excited state occurs prior to the hydrogen shift, making the hydrogen shift a key mechanistic event.

\section{Nanosecond Transient Absorption Studies}

To provide further insight into possible mechanisms for this reaction, we have studied this system using nanosecond laser flash photolysis with transient absorption. Briefly, samples were excited at $355 \mathrm{~nm}$ with an $8 \mathrm{~ns}$ pulse at $10 \mathrm{~Hz}$. On excitation of 1a, a transient spectrum with an absorption $\lambda_{\max }$ of 480 $\mathrm{nm}$ is observed (Figure 4). The transient absorption signal decayed in a single exponential with a lifetime $(\tau)$ of $930 \mathrm{~ns}$ in degassed methanol. Similar transients are seen from a number of structures (Table 1). In all cases we have observed that the products formed in the laser experiments are the same as in the steady-state photolysis. In air-equilibrated solutions, the same transient is observed, but in all cases the lifetime is in the 100 -200 ns range, consistent with diffusional quenching by oxygen. The observed transient is also quenched by amine-based quenchers. Considering the parent, 1a, in the presence of 10 $\mathrm{mM}$ triethylamine (TEA), an initial decay with a lifetime of $310 \mathrm{~ns}$ is seen, compared to $930 \mathrm{~ns}$ in the absence of TEA. As shown in Table 1 , there is a considerable variation in lifetime $(\tau)$ for the $480 \mathrm{~nm}$ transient. For the simple hydrocarbon systems (methyl, ethyl, isopropyl, benzyl), the transient lifetime tracks the product quantum yield measured in bulk, with the benzyl transient being significantly shorter lived than the methyl. As in the bulk photolysis, a significant KIE is seen for the transient lifetime for the benzyl compound (1e vs. 1e-d $\mathbf{d}_{2}$ ). However, a minimal KIE is seen for the methyl compound (1a vs. 1a-d $\mathbf{d}_{3}$.

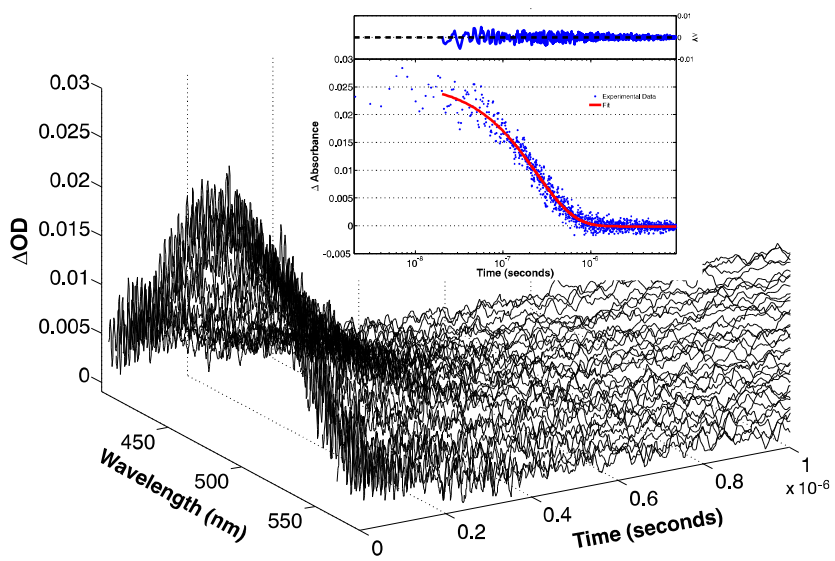

Figure 4. Transient absorption spectrum of 1a observed upon laser flash photolysis at $355 \mathrm{~nm}$ in aerated acetonitrile. Inset: single exponential fit (red) of transient decay at $480 \mathrm{~nm}$.

\section{Triplet Sensitization/Quenching Studies}

Excitation of simple quinones typically produces a triplet state with near unit efficiency. ${ }^{23,24}$ The present system, however, is significantly perturbed, electronically by the sulfur substituent and geometrically by the bulky trimethyl lock system. The long lifetime of the transient from the flash photolysis studies, and the fact that it is quenched efficiently by oxygen, suggest that the transient is a triplet. However, in steady-state photolysis studies, oxygen has only a small effect on the quantum yield. We have undertaken several studies to probe the role and nature of the triplet state in the photoreaction.

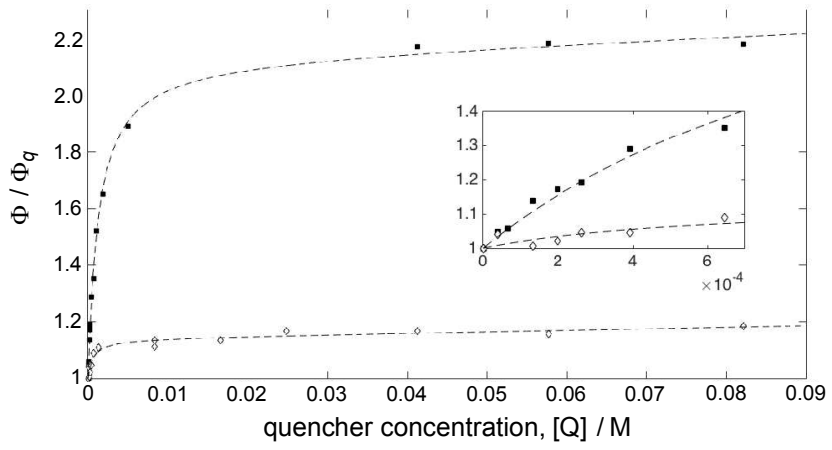

Figure 5. Stern-Volmer plot for the quenching of the quantum yield for disappearance of 1a (white diamonds) and 1e (black squares) by diethylaniline. Dotted curves are multivariable regression fits to Eq. 2. Inset is the low concentration region of the plot.

We initially considered the impact of triplet quenchers on the overall process, and obtained clean quenching with diethylaniline. Quenching by diethylaniline likely occurs through reversible electron transfer, as has been previously shown in similar systems. $^{45}$ Shown in Figure 5 are Stern-Volmer (SV) plots for photoreaction of the methyl (1a) and benzyl (1e) compounds, where $\Phi_{q}$ is defined as the quantum yield in the presence of quencher. At low concentrations of diethylaniline (up to $\sim 1 \mathrm{mM}$ ), roughly linear SV behavior is observed (Figure 5 , inset). However, at higher concentrations (up to $\sim 100$ $\mathrm{mM}$ ), the SV plot deviates from linearity and plateaus. This indicates that the photoreaction proceeds through two different pathways, one being much more efficiently quenched than the other. We have assigned the less and more quenchable portions of the photoreaction to those that occur through the singlet $\left(\Phi^{S}\right)$ and triplet $\left(\Phi^{T}\right)$ state, respectively. The sum of these pathways describes the overall quantum yield (Eq. 1).

$$
\begin{aligned}
\Phi & =\Phi^{\mathrm{S}}+\Phi^{\mathrm{T}} \\
\frac{\Phi}{\Phi_{q}} & =\frac{\left(1+K_{S V}^{S}[Q]\right)\left(1+K_{S V}^{T}[Q]\right)}{1+\frac{K_{S V}^{T}[Q]}{1+\left(\Phi^{T} / \Phi^{S}\right)}}
\end{aligned}
$$

Eq. 2 has been previously derived to describe the variation in quantum yield when there are two quenchable pathways. ${ }^{38}$ The expression includes two SV quenching constants, $K_{S V}^{S}$ and $K_{S V}^{T}$, for the singlet and triplet pathways, and the ratio of their quantum yields $\left(\Phi^{T} / \Phi^{S}\right)$. A regression analysis of Eq. 2 results in the fits shown in Figure 5. This analysis has been performed for key substrates, producing values of $\Phi^{S}$ and $\Phi^{T}$ after insertion of the determined ratio $\Phi^{T} / \Phi^{S}$ into Eq. 1 (Table 1). As expected, the triplet pathways of $\mathbf{1 a}$ and $\mathbf{1 e}$ are efficiently quenched, with similar $K_{S V}^{T}$ values of 1800 and $1700 \mathrm{M}^{-1}$, respectively. In contrast, the singlet states are negligibly quenched with $K_{S V}^{S}$ values of 0.45 and $0.52 \mathrm{M}^{-1}$. These two compounds are found to differ largely in their ratios of singlet to triplet reactivity, $\Phi^{T} / \Phi^{S}$. The calculated ratios reveal that, for $1 \mathrm{a}$, the reaction proceeds $88 \%$ through the singlet and $12 \%$ through the triplet. For 1e, the corresponding singlet and triplet values are $47 \%$ and $53 \%$. If we assume that the transient observed in laser flash photolysis is the triplet, we can use the transient lifetimes $(\tau)$ and $K_{S V}^{T}$ values to obtain $\mathrm{k}_{\mathrm{q}}$, the second- 
order rate constant for quenching. We find $\mathrm{k}_{\mathrm{q}}$ to be $10 \times 10^{9} \mathrm{M}^{-}$ ${ }^{1} \mathrm{~s}^{-1}$ and $2 \times 10^{9} \mathrm{M}^{-1} \mathrm{~s}^{-1}$ for $1 \mathrm{a}$ and $1 \mathrm{e}$, respectively, which are similar to diffusion-controlled values in methanol, where $\mathrm{k}_{\text {diff }}$ $=1.2 \times 10^{10} \mathrm{M}^{-1} \mathrm{~s}^{-1}$.

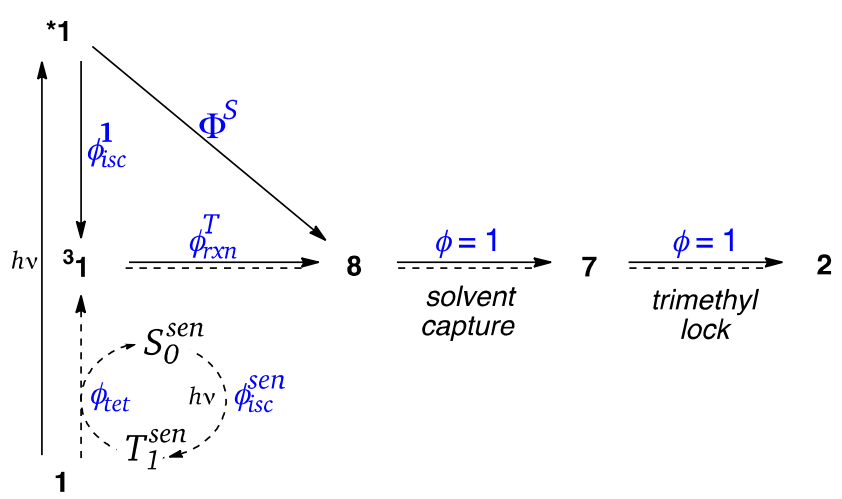

Figure 6. Processes that contribute to the direct (solid) and sensitized (dashed) quantum yields for the disappearance of 1 upon excitation at $420 \mathrm{~nm}$. Nonproductive processes have been omitted for clarity.

The results from the quenching experiments reveal that the overall quantum yield $(\Phi$, Table 1$)$ measured in the steadystate photolysis has components from both the singlet $\left(\Phi^{S}\right)$ and triplet $\left(\Phi^{T}\right)$ pathways (Eq. 1). A minimal model describing the relevant steps that contribute to these pathways is shown in Figure 6, where nonproductive processes have been omitted for clarity. According to this model, the quantum yield from the singlet $\left(\Phi^{S}\right)$ due to direct photolysis of the quinone is defined by the pathway $1 \rightarrow * 1 \rightarrow 8 \rightarrow 7 \rightarrow 2$. The conversion of $\mathbf{8} \rightarrow \mathbf{7} \rightarrow \mathbf{2}$ occurs with unit efficiency, as evidenced by the clean formation of product and the irreversibility of the hydrogen-shift. $\Phi^{S}$ is therefore simply defined by the efficiency of $* 1 \rightarrow 8$.

Likewise, disappearance of quinone through the triplet state $\left(\Phi^{T}\right)$ upon direct photolysis is defined by the pathway $\mathbf{1} \rightarrow *^{*}$ $\rightarrow^{3} \mathbf{1} \rightarrow \mathbf{8} \rightarrow \mathbf{7} \rightarrow \mathbf{2}$. The quantum yield for this process, given by Eq. 3, is constructed as the product of the contributing efficiencies, namely $\phi_{i s c}^{\mathbf{1}}$, the efficiency of triplet formation via intersystem crossing, and $\phi_{r x n}^{T}$, conversion of triplet to zwitterion 8.

$$
\Phi^{T}=\phi_{i s c}^{1} \cdot \phi_{r x n}^{T}
$$

We sought to explore the nature of this triplet pathway in more detail through the use of triplet sensitizers. Many efforts to employ sensitizers were either ineffective (acetophenone, benzophenone, methylene blue) or produced undesired side products (biacetyl, naphthalene, anthracene, rose bengal). However, thioxanthone produced clean and consistent results. Also shown in Figure 6 are processes that contribute to the sensitized quantum yield for the disappearance of $\mathbf{1}$. The pathway begins with excitation and intersystem crossing of the sensitizer, $S_{0}^{\text {sen }} \rightarrow T_{1}^{\text {sen }}$, followed by bimolecular triplet energy transfer to the quinone, $T_{1}^{\text {sen }}+\mathbf{1} \rightarrow S_{0}^{\text {sen }}+{ }^{3} \mathbf{1}$. Conversion of the triplet quinone to product then proceeds normally $\left({ }^{3} \mathbf{1} \rightarrow \mathbf{8} \rightarrow 7\right.$ $\rightarrow 2$ ). The quantum yield for this pathway $\left(\Phi_{s e n}\right)$ is given by the product shown in Eq. 4 , where $\phi_{i s c}^{\text {sen }}$ is the efficiency of intersystem crossing for the sensitizer, and $\phi_{t e t}$ is the efficiency of triplet energy transfer. The efficiency of triplet energy transfer depends upon the concentration of quinone, [1], and is described by Eq. 5, where $\tau^{\text {sen }}$ is the intrinsic lifetime of the triplet sensitizer, and $k_{t e t}[\mathbf{1}]$ and $k_{n p}[\mathbf{1}]$ are pseudo-first order rate constants for deactivation of the sensitizer through productive and nonproductive collisions with quinone, respectively.

$$
\begin{aligned}
\Phi_{\text {sen }} & =\phi_{\text {isc }}^{\text {sen }} \cdot \phi_{\text {tet }} \cdot \phi_{\text {rxn }}^{T} \\
\phi_{\text {tet }} & =\frac{k_{\text {tet }}[\mathbf{1}]}{1 / \tau^{\text {sen }}+k_{t e t}[\mathbf{1}]+k_{n p}[\mathbf{1}]}
\end{aligned}
$$

Insertion of Eq. 5 into Eq. 4, and taking the inverse reveals a double-reciprocal linear relationship between the sensitized quantum yield $\left(\Phi_{\text {sen }}\right)$ and quinone concentration ([1]) (Eq. 6), where the product $k_{\text {tet }} \bullet \tau^{\text {sen }}$ is recognized as a Stern-Volmer constant $K_{S V}^{t e t}$ for the productive quenching of the triplet sensitizer by 1 . The reciprocal of the y-intercept in Eq. 6 is designated as $\Phi_{\text {sen }}^{\text {lim }}$ (Eq. 7) and describes the sensitized quantum yield for the disappearance of $\mathbf{1}$ in the limit where deactivation of the triplet sensitizer occurs exclusively through collisions with the quinone. Similar analyses have been presented previously. $^{47}$

$$
\begin{aligned}
\frac{1}{\Phi_{\text {sen }}} & =\frac{1}{\phi_{i s c}^{\text {sen }} \cdot \phi_{r x n}^{T}}\left(\frac{1}{K_{S V}^{t e t}} \cdot \frac{1}{[1]}+\frac{k_{t e t}+k_{n p}}{k_{t e t}}\right) \\
\Phi_{\text {sen }}^{\text {lim }} & =\phi_{i s c}^{\text {sen }} \cdot \phi_{\mathrm{r} x n}^{T} \cdot \frac{k_{t e t}}{k_{t e t}+k_{n p}}
\end{aligned}
$$

Representative double-reciprocal plots are shown in Figure 7 for 1a, 1e, and the deuterated anologs $\mathbf{1} \mathbf{a}-\mathbf{d}_{\mathbf{3}}$ and $\mathbf{1 e - \mathbf { d } _ { 2 }}$. Determination of $\Phi_{\text {sen }}^{\text {lim }}$ from the fit is accomplished by averaging three independent samples. Although the standard deviation in $\Phi_{\text {sen }}^{\text {lim }}$ is consistently less than $10 \%$ (Figure 7, error bars), we note that the slope in the double-reciprocal plots is unexpectedly sensitive to the concentration of thioxanthone. This fact is demonstrated explicitly for $\mathbf{1 a}$, where three samples containing 1,2 , and $3 \mathrm{mM}$ thioxanthone resulted in incremental shifts in the slope. The y-intercept, however, is clearly unaffected. This kind of behavior has been seen before, ${ }^{47}$ and it was not probed further.

If we assume that each quenching event of the sensitizer leads to triplet energy transfer, then $k_{-}$tet $/\left(k_{-} t e t+k_{-} n p\right)=1$. This, combined with the known efficiency of intersystem crossing for thioxanthone $\left(\phi_{i s c}^{\text {sen }}\right)$ of $0.56,{ }^{48,49}$ allows the determination of $\phi_{r x n}^{T}$ values, which are reported in Table 1 . These values represent lower limits, given the assumption above. In general, $\phi_{r x n}^{T}$ is much larger than $\Phi$, suggesting that the triplet state is not efficiently generated by direct photolysis. Substitution of $\phi_{r x n}^{T}$ into Eq. 3 permits calculation of the efficiency for intersystem crossing by the quinone $\left(\phi_{i s c}^{\mathbf{1}}\right.$, Table 1). Although quinones typically form triplets with high yields, ${ }^{23}$ the efficiencies observed in this system do not exceed $10 \%$, likely due to the electronic and steric effects of the sulfide and trimethyl-lock substituents, respectively.

The first-order rate constants for formation of $\mathbf{8}$ from the triplet state $\left(k_{r \times n}^{T}\right)$ can be calculated using Eq. 8 if we assume that the triplet considered here is the transient observed in laser flash photolysis. Although the results, collected in Table 1 , reflect broad trends in bond dissociation energy (BDE) with the simple alkyl substituents $\mathbf{1 a}-\mathbf{1 c}$ reacting slower than benzylic substituent 1e, the rates are clearly complicated by other factors. For instance, the fact that the methyl (1a) and isopropyl (1c) derivatives have essentially the same rate constant cannot be explained using simple BDE arguments alone. 


$$
\phi_{r x n}^{T}=k_{r x n}^{T} \cdot \tau
$$

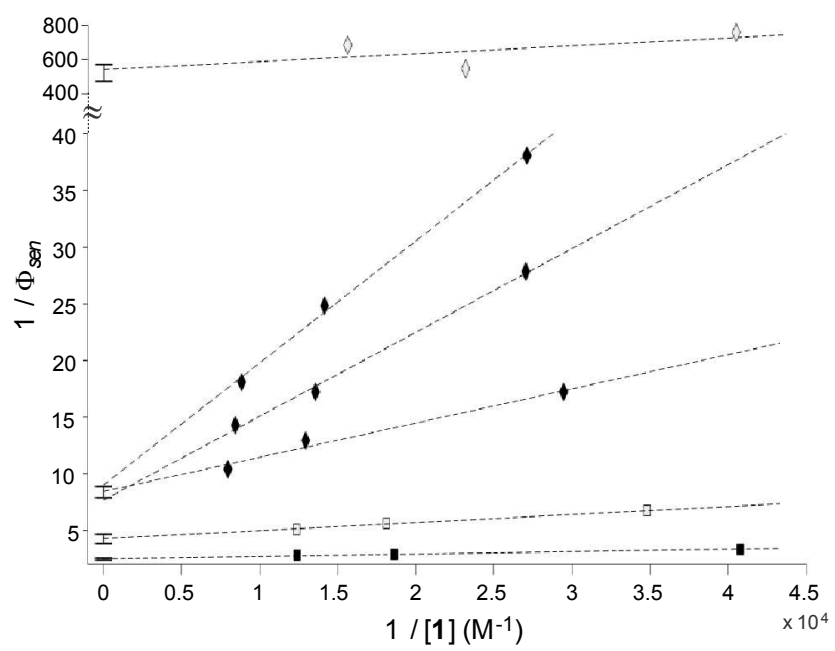

Figure 7. Double reciprocal plots for the sensitized photolysis of 1 by thioxanthone in degassed methanol. 1e (black squares), 1e-d $\mathrm{d}_{2}$ (white squares), 1a (black diamonds), 1a-d 3 (white diamonds). Dotted lines are linear fits; error bars are the standard deviation in the $y$-int for three independent samples. Three samples of 1a using different concentrations of thioxanthone are shown to demonstrate that the slope, but not the $y$-intercept, is affected.

Significant isotope effects on the sensitized quantum yield $\left(\phi_{r x n}^{T}\right)$ are also observed in Figure 7 and Table 1. For the $\mathbf{1 e} / \mathbf{1}$ e-d $\mathbf{d}_{2}$ system, a magnitude of 1.7 , given by the ratio $\phi_{H} / \phi_{D}$ is similar to the magnitude of 2.5 observed in direct photolysis. Application of Eq. 8 to these data reveal a large normal $\operatorname{KIE}\left(k_{H} / k_{D}\right)$ of 7 . A similar analysis shows that the methyl analogue, $\mathbf{1 a} / \mathbf{1} \mathbf{a}-\mathbf{d}_{3}$, experiences a very large sensitized product isotope effect $\left(\phi_{H} / \phi_{D}\right)$ of 70 , compared to a direct photolysis isotope effect of 4 . In particular, we find that the deuterated analog $\left(\mathbf{1} \mathbf{a}-\mathbf{d}_{\mathbf{3}}\right)$ is very inefficient in the sensitized photolysis (Figure 7), with a quantum yield $\left(\phi_{r x n}^{T}\right)$ of 0.03 , comparable to the efficiency for direct photolysis $(\Phi)$. Calculation of the KIE for $\mathbf{1 a} / \mathbf{1} \mathbf{a}-\mathbf{d}_{\mathbf{3}}$ from the transient lifetimes and Eq. 8 yields a value $\left(k_{H} / k_{D}\right)$ of 70 .

\section{Mechanistic Interpretation}

Based on the accumulated evidence, we argue that the most plausible mechanism is the one outlined in Figure 8 (a more complete version of the ionic path in Scheme 2). The penultimate intermediate is the zwitterion $\mathbf{8}$; once it is formed, product formation involves solvent capture and subsequent trimethyl lock ring closure. That $\mathbf{8}$ proceeds with high efficiency $(>95 \%)$ to the final products is evidenced by the stereochemical labeling studies. Formation of $\mathbf{8}$ can occur in both the singlet and the triplet manifolds. For the simple alkyl substrates, product formation is dominated by the singlet pathway, as evidenced by a large contribution of $\Phi^{\mathrm{S}}$ to the overall quantum yield, $\Phi$ (Table 1). The more efficient reaction for the benzylic substrates includes a more substantial contribution from the triplet pathway.

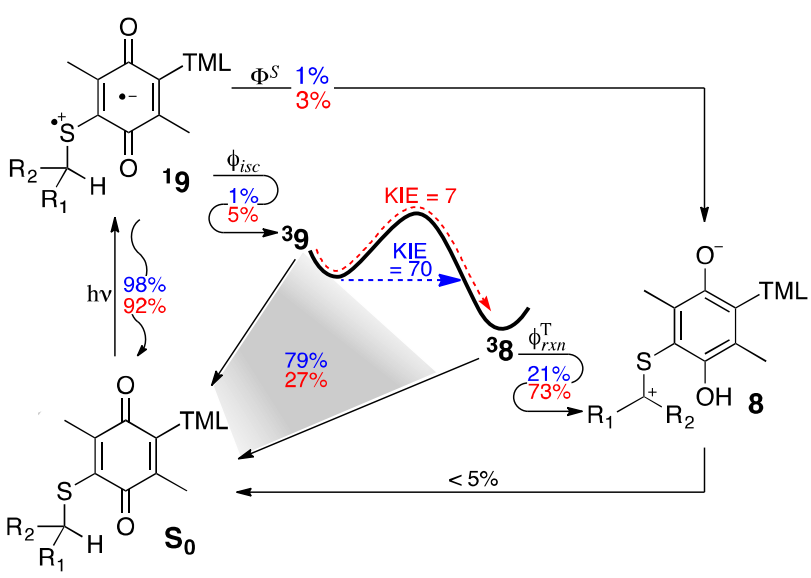

Figure 8. The overall photochemical transformation and efficiencies for the conversion of 1 a $(R=H$; blue) and $1 \mathrm{e}(\mathrm{R}=\mathrm{Ph}$; red) to the zwitterion 8. The ester side chain is abbreviated TML.

Based on several lines of evidence, we consider the initial excited state to be the charge transfer state, ${ }^{1} \mathbf{9}$, as in Figure 8, rather than a simple $n, \pi^{*}$ state. The broad visible absorption band observed in these compounds (Figure 3 ) is common for benzoquinones bearing sulfide or amino groups, and is indicative of charge-transfer. ${ }^{29}$ The dominant fate of ${ }^{1} \mathbf{9}$ is nonemissive return to the ground state ( $>90 \%$ efficiency), again in contrast to simpler benzoquinones, which typically undergo intersystem crossing to the triplet with near unit efficiency. ${ }^{23}$

The triplet state does form with low efficiency, and again it is not like the triplet of a typical benzoquinone. Shown in Figure 9 are spin-density plots for relevant species computed using DFT M06/6-311++G**, where the trimethyl lock side chain is not displayed for clarity but is included in the calculation. The parent benzoquinone is a conventional $\mathrm{n}, \pi^{*}$ triplet state (consistent with experiment) ${ }^{50}$ demonstrated by significant spin density on the oxygen n-orbitals orthogonal to the $\pi$-system (Figure 9A). In sharp contrast, the triplet state of $\mathbf{1}$ is wellrepresented by the charge-transfer structure, ${ }^{3} \mathbf{9}$, with no spin density on the oxygen n-orbitals, indicating that they are doubly-occupied. There is also significant spin-density on sulfur (Figure 9B).

Typically, when given the opportunity, excited states of simple benzoquinones will readily undergo $\gamma$-hydrogen abstraction. ${ }^{36,37,51}$ The trimethyl lock unit of the present system possesses eight $\gamma$-hydrogens, but we see no products suggesting that hydrogen abstraction occurs from that unit. In contrast, compound $\mathbf{6}$, lacking sulfur, undergoes efficient abstraction of the trimethyl lock hydrogens, but 1d, possessing a t-butyl substituent on sulfur, is remarkably photostable. These observations suggest formation of a charge transfer excited state that, in both the singlet and triplet states, has a reactivity pattern that is significantly different from that of a simple benzoquinone. While the charge transfer excited state converts to products with low efficiency, apparently it also suppresses the typical reactivity of benzoquinones, and so product formation is very clean. 


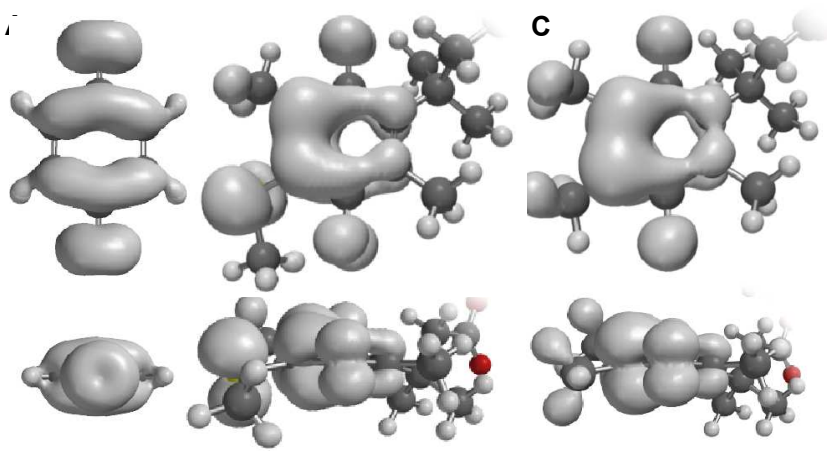

Figure 9. Spin-density plots for the triplet state of (A) benzoquinone,(B) 1a, and (C) 6 computed using M06/6-311++G**. The trimethyl lock side chain is not displayed for clarity.

We conclude that the transient observed in the laser flash photolysis experiments is the triplet state, ${ }^{\mathbf{3}} \mathbf{9}$. That the transient is a triplet is supported by quenching data for both oxygen and diethylaniline, and by its long lifetime. ${ }^{52}$ That this triplet is on the reaction pathway is supported by several lines of evidence. Product formation is very clean in the transient absorption experiments. Additionally, a significant KIE for the transient lifetime indicates that the transient is a species that undergoes a hydrogen-shift reaction. Also, to a considerable extent, the lifetime of the transient tracks with the quantum yield of the bulk reaction (Table 1). We would not expect a perfect correlation, because both singlet and triplet paths are involved in the bulk photolysis. Based on its reactivity pattern and the computations noted above, we conclude that this triplet has considerable charge transfer character, unlike a typical Norrish II triplet. Also, for the alkyl substrates, reaction from the triplet is unusually slow $\left(k_{r x n}^{T} \sim 10^{5} \mathrm{~s}^{-1}\right.$, Table 1$)$ compared to typical $\gamma$-hydrogen shifts, which have rate constants greater than $10^{7} \mathrm{~s}^{-1}$ for typical ketones ${ }^{53}$ and simple benzoquinones. ${ }^{51}$ We interpret this to reflect the decreased $n, \pi^{*}$ character in the triplet state of the structures studied here. Since $\gamma$-hydrogen shifts are typically initiated by the electrophilic oxygen that is characteristic of an $n, \pi^{*}$ state, ${ }^{53} \mathbf{9}$ is expected to react more slowly through this type of mechanism. Electron-donating substituents are known to preferentially stabilize the lowest $\pi, \pi^{*}$ triplet state of quinones, ${ }^{23,54}$ and the substituents of $\mathbf{1}$, especially the sulfide, are likely to exhibit this effect in ${ }^{\mathbf{3}} \mathbf{9}$. The triplet state of $\mathbf{6}$, lacking sulfur, is also predicted to possess a high degree of $\pi, \pi^{*}$ character (Figure 9C), suggesting that alkyl substituents alone may have a substantial effect on the electronics of the quinone. While this could be due to geometric distortion brought on by the trimethyl lock, some unstrained quinones bearing alkyl substituents are also known to have lowest $\pi, \pi^{*}$ triplet states. ${ }^{50}$

In the present system, strong electron-withdrawing groups, such as $p$-nitrobenzyl (1h) and methyl acetate (1i), result in considerably lower quantum yields for quinone consumption and yield a complex mixture of photolysis products. Again, this is consistent with the presence of an ionic structure on the pathway, as it would be destabilized by electron withdrawing substituents due to the significant positive charge on the carbon attached to sulfur.

Although the triplet is formed in low yields, it proceeds to the product much more efficiently than the excited singlet. For benzylic derivatives, values of $\phi_{r x n}^{T}$ can approach unity. The effect of substitution on the triplet photoreaction as a whole is complicated. The $p$-chlorobenzyl derivative (1g) is more efficient than the benzyl derivative (1e) not because the hydrogenshift is more favorable, but because the rate of nonproductive triplet decay is lessened. In Figure 8 we show the triplet pathway as ${ }^{3} \mathbf{9} \rightarrow{ }^{3} \mathbf{8} \rightarrow \mathbf{8}$. However, a direct conversion ${ }^{3} \mathbf{9} \rightarrow \mathbf{8}$ seems possible. As noted above, the reaction is slower than a typical hydrogen transfer. Additionally, since the triplet accounts for less than $10 \%$ of the excited states, the stereochemical labeling studies do not establish whether nonproductive triplet decay occurs directly from ${ }^{3} \mathbf{9}$ or through reverse hydrogen shift from ${ }^{\mathbf{3}}$.

Another intriguing feature of this system is the collection of KIEs seen. The macroscopic quantum yield shows a moderate isotope effect. More interestingly, combining the transient absorption spectroscopy with the sensitized photolysis studies allows a determination of the true KIE, $\mathrm{k}_{\mathrm{H}} / \mathrm{k}_{\mathrm{D}}$, for the triplet. For the benzyl system, $\mathbf{1 e} / \mathbf{1} \mathbf{e}-\mathbf{d}_{2}$, a value of 7 is observed, which is relatively large for a reaction of this sort. Remarkably, the parent methyl system, $\mathbf{1 a} / \mathbf{1} \mathbf{a}-\mathbf{d}_{\mathbf{3}}$, gives a $\mathrm{k}_{\mathrm{H}} / \mathrm{k}_{\mathrm{D}}$ of 70 (Table 1). This effect is not evident in transient lifetime data because the dominant decay path for the $\mathbf{1 a} / \mathbf{1 a}-\mathbf{d}_{3}$ triplet is return to $\mathrm{S}_{0}$. Such a large KIE strongly implies tunneling in the hydrogen shift for this system. While $\gamma$-hydrogen shifts by triplet ketones are generally understood to be activated processes, certain constrained systems have been designed to probe for tunneling at low temperature. ${ }^{55,56}$ The tunneling rates observed in those systems by deuterium are found to be similar to $k_{r x n}^{T}$ for $\mathbf{4 a - d _ { 3 }}\left(\sim 10^{3} \mathrm{~s}^{-1}\right)$, indicating that nonclassical effects may be observable for this slow reaction even at room temperature.

\section{Conclusions}

We describe mechanistic studies of a new reaction that allows rapid photochemical decaging of a wide range of structures using the well-established trimethyl lock lactonization process. Key to the development of this system was the discovery of an effective phototrigger based on an intramolecular redox reaction of benzoquinones bearing a sulfide substituent. Our results indicate that the process begins with photoinduced electron transfer, followed by a critical and irreversible hydrogen shift that ultimately results in two electron reduction to the hydroquinone. The nucleophilic hydroquinone oxygen is then capable of undergoing rapid, thermal trimethyl lock lactonization with release of the caged compound. Both singlet and triplet pathways are viable, with the latter proceeding to product much more efficiently.

Given our mechanistic conclusions, many strategies for extending the excitation wavelength can be envisioned, as photoinduced electron transfer is a heavily studied and wellunderstood process. Also, the modular synthesis of these compounds allows the substituent on sulfur to be readily varied, allowing the introduction of groups that impact solubility, cell permeability, and biodistribution in general. Further studies along these lines are underway.

\section{ASSOCIATED CONTENT}

\section{Supporting Information}

Synthetic procedures and characterization data, procedures for quantum yield determination and transient absorption experiments (PDF) are available free of charge on the ACS Publications website. 


\section{AUTHOR INFORMATION}

Corresponding Author

*dadougherty@caltech.edu

\section{Notes}

The authors declare no competing financial interest.

\section{ACKNOWLEDGMENT}

This work was supported by the W. M. Keck Foundation. Additional support was provided by the Arnold and Mabel Beckman Foundation through the Caltech Beckman Institute Laser Resource Center. We thank Jay Winkler for technical assistance and helpful discussions, and Scott Virgil for assistance with studies of compound $\mathbf{1 n}$.

\section{REFERENCES}

(1) Walton, D.; Dougherty, D.A., submitted.

(2) Levine, M. N.; Raines, R. T. Chem. Sci. R. Soc. Chem. 2010 2012, 3 (8), 2412.

(3) Greenwald, R. B.; Choe, Y. H.; Conover, C. D.; Shum, K.; Wu, D.; Royzen, M. J. Med. Chem. 2000, 43, 475.

(4) Gomes, P.; Vale, N.; Moreira, R. Molecules 2007, 12, 2484.

(5) Pelliccioli, A. P.; Wirz, J. Photochem. Photobiol. Sci. 2002, 1,441 .

(6) Klán, P.; Šolomek, T.; Bochet, C. G.; Blanc, A.; Givens, R.; Rubina, M.; Popik, V.; Kostikov, A.; Wirz, J. Chem. Rev. 2013, 113 (1), 119.

(7) Görner, H. Photochem. Photobiol. 2006, 82, 71.

(8) Kavarnos, G. J.; Turro, N. J. Chem. Rev. 1986, 86, 401.

(9) Görner, H. Photochem. Photobiol. 2003, 78, 440.

(10) Scheerer, R.; Graetzel, M. J. Am. Chem. Soc. 1977, 99, 865. 268.

(11) Baxter, I.; Phillips, W. R. J. Chem. Soc. [Perkin 1] 1973,

(12) Bruce, J. M. Q. Rev. Chem. Soc. 1967, 21, 405.

(13) Iwamoto, H.; Takuwa, A.; Hamada, K.; Fujiwara, R. J. Chem. Soc. [Perkin 1] 1999, 575.

(14) Kallmayer, H.-J.; Fritzen, W. Pharmazie 1994, 49, 412.

(15) Shi, M.; Yang, W.-G.; Wu, S. J. Photochem. Photobiol. Chem. 2007, 185, 140.

(16) Chen, Y.; Steinmetz, M. G. J. Org. Chem. 2006, 71, 6053.

(17) Chen, Y.; Steinmetz, M. G. Org. Lett. 2005, 7, 3729.

(18) Jones; Qian, X. J. Phys. Chem. A 1998, 102, 2555.

(19) Falci, K. J.; Franck, R. W.; Smith, G. P. J. Org. Chem. 1977, $42,3317$.

(20) Giles, R. G. F. Tetrahedron Lett. 1972, 13, 2253.

(21) Cameron, D. W.; Giles, R. G. F. J. Chem. Soc. C Org. 1968, 1461.

(22) Cameron, D. W.; Giles, R. G. F. Chem. Commun. Lond. $1965,573$.
(23) Barbafina, A.; Latterini, L.; Carlotti, B.; Elisei, F. J. Phys. Chem. A 2010, 114, 5980.

(24) Hubig, S. M.; Bockman, T. M.; Kochi, J. K. J. Am. Chem. Soc. 1997, 119, 2926.

(25) Trommsdorff, H. P. J. Chem. Phys. 1972, 56, 5358.

(26) Asundi, R. K.; Singh, R. S. Nature 1955, 176, 1223.

(27) Bridge, N. K.; Porter, G. Proc. R. Soc. Lond. Math. Phys. Eng. Sci. 1958, 244, 259.

(28) Orgel, L. E. Trans. Faraday Soc. 1956, 52, 1172.

(29) Braude, E. A. J. Chem. Soc. Resumed 1945, 490.

(30) Bridge, N. K.; Porter, G. Proc. R. Soc. Lond. Math. Phys. Eng. Sci. 1958, 244, 276. 3782 .

(31) Orlando, C. M.; Bose, A. K. J. Am. Chem. Soc. 1965, 87,

(32) Orlando, C. M.; Mark, H.; Bose, A. K.; Manhas, M. S. J. Am. Chem. Soc. 1967, 89, 6527.

(33) Orlando, C. M.; Mark, H.; Bose, A. K.; Manhas, M. S. J. Org. Chem. 1968, 33, 2512.

(34) Baxter, I.; Mensah, I. A. J. Chem. Soc. C Org. 1970, 2604.

(35) King, T. J.; Forrester, A. R.; Ogilvy, M. M.; Thomson, R. H. J. Chem. Soc. Chem. Commun. 1973, 844.

(36) Kraus, G. A.; Wu, Y. J. Am. Chem. Soc. 1992, 114, 8705.

(37) Görner, H. J. Photochem. Photobiol. Chem. 2005, 175, 138.

(38) Klán, P.; Wirz, J. In Photochemistry of Organic Compounds; John Wiley \& Sons, Ltd, West Sussex, UK, 2009; pp 73-135.

(39) Hu, S.; Neckers, D. C. J. Org. Chem. 1997, 62, 755.

(40) Griller, D.; Ingold, K. U. Acc. Chem. Res. 1980, 13, 317.

(41) Wagner, P. J.; Liu, K. C. J. Am. Chem. Soc. 1974, 96, 5952.

(42) Small, R. D.; Scaiano, J. C. J. Phys. Chem. 1977, 81, 2126.

(43) Engel, P. S.; Keys, D. E. J. Am. Chem. Soc. 1982, 104, 6860.

(44) Castellino, A. J.; Bruice, T. C. J. Am. Chem. Soc. 1988, 110, 7512 .

(45) Amouyal, E.; Bensasson, R. J. Chem. Soc., Faraday Trans. 1 1977, 73, 1561 .

(46) Klán, P.; Wirz, J. In Photochemistry of Organic Compounds; John Wiley \& Sons, Ltd, West Sussex, UK, 2009; pp 467-470.

(47) Balzani, V.; Ballardini, R.; Gandolfi, M. T.; Moggi, L. J. Am. Chem. Soc. 1971, 93, 339.

(48) Allonas, X.; Ley, C.; Bibaut, C.; Jacques, P.; Fouassier, J. P. Chem. Phys. Lett. 2000, 322, 483.

(49) Burget, D.; Jacques, P. J. Lumin. 1992, 54, 177.

(50) Kemp, D. R.; Porter, G. Proc. R. Soc. Lond. Math. Phys. Eng. Sci. 1971, 326, 117.

(51) Görner, H. J. Photochem. Photobiol. Chem. 2004, 165, 215.

(52) Barbafina, A.; Elisei, F.; Latterini, L.; Milano, F.; Agostiano, A.; Trotta, M. Photochem. Photobiol. Sci. 2008, 7, 973.

(53) Wagner, P. J. Acc. Chem. Res. 1971, 4, 168.

(54) Amouyal, E.; Bensasson, R.; Land, E. J. Photochem. Photobiol. 1974, 20, 415.

(55) Garcia-Garibay, M. A.; Gamarnik, A.; Bise, R.; Pang, L.; Jenks, W. S. J. Am. Chem. Soc. 1995, 117, 10264. 
(56) Johnson, B. A.; Kleinman, M. H.; Turro, N. J.; Garcia-

1

2

3

4

5

6

7

8

9

10

11

12

13

14

15

16

17

18

19

20

21

22

23

24

25

26

27

28

29

30

31

32

33

34

35

36

37

38

39

40

41

42

43

44

45

46

47

48

49

50

51

52

53

54

55

56

57

58

59

60 


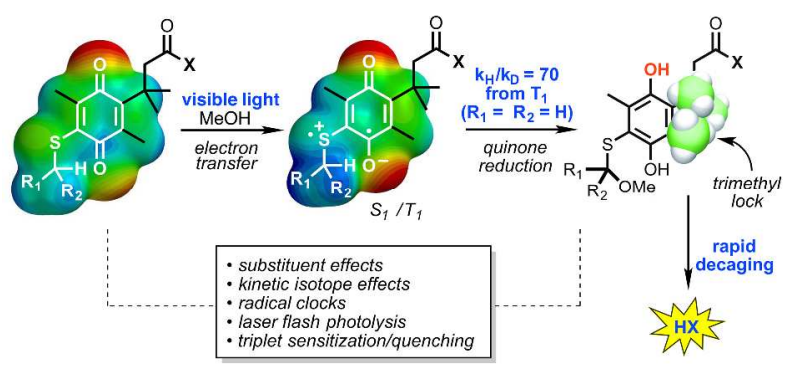

